\title{
Robust Maximum-Likelihood Position Estimation in Scintillation Cameras
}

\author{
Jeffrey A. Fessler, W. Leslie Rogers, and Neal H. Clinthorne \\ Division of Nuclear Medicine, University of Michigan
}

\begin{abstract}
The classical maximum-likelihood (ML) estimator for the position of a scintillation event in a gamma camera, as derived by Gray and Macovski in 1976, requires exact knowledge of the light-spread function (LSF) of each photomultiplier tube. In practice, one must determine each LSF from noisy measurements corrupted by Poisson noise, quantization error, and electronic noise and bias. Since the ML position estimator involves derivatives of each LSF, even small measurement errors can result in degraded estimator performance. In this paper we derive a robust $\mathrm{ML}$ position estimator that accounts for the statistical uncertainty in LSF measurements. The form of the robust estimator diminishes contributions from the tails of the LSF, where the relative measurement errors are the largest.
\end{abstract}

\section{INTRODUCTION}

Position-sensitive scintillation cameras play a crucial role in nuclear medicine; they are used to form conventional planar images, as well as to collect projection measurements for reconstruction into cross-sectional images of radio-isotope distributions. When a decay-produced gamma photon strikes a position sensitive detector, the scintillation crystal produces visible photons that are sensed by a photomultiplier tube (PMT) array. The PMT output currents are combined electronically to form an estimate of the position of the scintillation event. The accuracy of the position estimator affects the spatial resolution of planar images, and also contributes to the effective resolution of tomographic images. Furthermore, systematic errors in position estimation can lead to artifacts in reconstructed images. Such artifacts were the impetus for this study.

There have been several position estimators proposed in the literature. The most common and straightforward approach is to use a centroid or trimmed-centroid estimator. The centroid estimate is suboptimal unless the light-spread function (LSF) (the mean PMT response as

\footnotetext{
*This work was supported in part by NCI Training Grant 5 T32 CA09015, and by a DOE Alexander Hollaender Postdoctoral Fellowship.

0-7803-0513-2/92\$0.3.00 @IEEE
}

a function of position) of each PMT has Gaussian shape The centroid estimate also disregards the statistical properties of scintillation cameras. This led Gray and Macovski [1] to propose applying the maximum-likelihood (ML) criterion for position estimation. Clinthorne and Rogers et al [2] implemented an ML estimator in hardware for SPECT, and demonstrated that the ML position estimator significantly outperforms the centroid position estimator and nearly achieves the Cramér-Rao (CR) lower bound. The implementation in [2] is based on separating the twodimensional position estimate into two one-dimensional estimation problems, and for simplicity we adopt that approach here as well.

The derivations in [1] and [2] are based on the assumption that each LSF is known exactly. In practice, one must determine each LSF from noisy measurements corrupted by Poisson noise, quantization error, and electronic noise and bias. This paper presents a new position estimator that is more robust in that it accounts for the Poisson noise in LSF measurements.

Before presenting the robust position estimator, we first review the derivation of the ordinary ML position estimator. Let $K$ be the number of PMTs, and let the output of the $k$ th PMT be denoted $y_{k}$. The PMT outputs have independent Poisson distributions:

$$
y_{k} \sim \operatorname{Poisson}\left(\varepsilon s_{k}(x)\right)
$$

where $s_{k}(x)$ denotes the LSF of the $k$ th PMT, $\varepsilon$ denotes the (unknown) gamma photon energy, and $x$ denotes the scintillation position. The purpose ${ }^{1}$ of a position estimator is to estimate $x$ given $y_{1}, \ldots, y_{K}$. The position log-likelihood is thus:

$$
L(x, \varepsilon)=\sum_{k=1}^{K}\left[-\varepsilon s_{k}(x)+y_{k} \log \left(\varepsilon s_{k}(x)\right)\right] .
$$

As derived in [2] (see also the next section), if each LSF $s_{k}(x)$ is known exactly, then one can analytically solve for the ML estimate of $\varepsilon$, and compute the ML position esti-

\footnotetext{
${ }^{1}$ Note that for systems with multiple energy windows, the parameter $\varepsilon$ is also important. Here we treat $\varepsilon$ as a nuisance parameter.
} 
mate for $x$ by maximizing:

$$
\sum_{k=1}^{K} y_{k}\left[\log \left(s_{k}(x)\right)-\log \left(\sum_{j=1}^{K} s_{j}(x)\right)\right] .
$$

In particular, one can implement the ML estimator [2] by searching for a zero-crossing of

$$
\sum_{k=1}^{K} y_{k}\left[\frac{\frac{\partial}{\partial x} s_{k}(x)}{s_{k}(x)}-\frac{\sum_{j=1}^{K} \frac{\partial}{\partial x} s_{j}(x)}{\sum_{j=1}^{K} s_{j}(x)}\right]
$$

as a function of $x$. If each LSF is known, then one can show [2] that the variance of this ML estimator is approximately

$$
\operatorname{Var}(\hat{x}) \approx\left[\sum_{k=1}^{K} \frac{\frac{\partial}{\partial x} s_{k}(x)}{s_{k}(x)}-\frac{\sum_{j=1}^{K} \frac{\partial}{\partial x} s_{j}(x)}{\sum_{j=1}^{K} s_{j}(x)}\right]^{-1},
$$

which nearly achieves the CR bound [2]:

$$
\operatorname{Var}(\hat{x}) \geq \sigma_{\mathrm{CR}}^{2}=\left[\sum_{k=1}^{K} \frac{\frac{\partial}{\partial x} s_{k}(x)}{s_{k}(x)}\right]^{-1}
$$

In practice, one must determine each LSF from noise corrupted calibration measurements. Since the ML position estimator (3) involves derivatives of each LSF, even small measurement errors can result in degraded performance. In this paper we propose a novel estimator that accounts for the statistical uncertainty in the LSF measurements.

\section{ROBUST POSITION ESTIMATOR}

In practice, one measures each LSF during a calibration procedure by translating a point source of gamma rays to a finite number of positions along the detector. Let the source positions be denoted $\left\{x_{m}\right\}_{m=1}^{M}$. While the source is at position $x_{m}$, the output of each PMT is observed for a finite time interval, producing $l_{k}\left(x_{m}\right)$, a noisy estimate of $s_{k}\left(x_{m}\right), m=1, \ldots, M, k=1, \ldots, K$. We assume these LSF measurements have independent Poisson distributions:

$$
\alpha l_{k}\left(x_{m}\right) \sim \operatorname{Poisson}\left(\alpha s_{k}\left(x_{m}\right)\right) .
$$

The parameter $\alpha$ reflects the accuracy of the measurements and is easily determined empirically using, for example, the jackknife estimate of variance [3]. Ideally, $\alpha$ would equal the number of scintillations that occur during each time interval. The assumption of independence is easily justified by fact that the LSF measurements are collected independently. The Poisson assumption reflects the fact that only a finite number of gamma events occur during the calibration time interval. However, the Poisson assumption ignores the electronic noise and bias as well as contributions from background events. We return to these considerations in the discussion section.
Given a scintillation event with PMT measurements $\left\{y_{k}\right\}_{k=1}^{K}$, our goal is to estimate its position $x$ while accounting for the uncertainty in $\left\{s_{k}(x)\right\}$. The natural statistical approach is to consider both $x$ and $\left\{s_{k}(x)\right\}$ to be unknown, and to maximize their joint log-likelihood with respect to both the LSF measurements and the PMT outputs. Under our assumptions for the distributions of $\left\{l_{k}\left(x_{m}\right)\right\}$, the log-likelihood of the LSF measurements is:

$$
L_{1}\left(\left\{\mathbf{s}\left(x_{m}\right)\right\}_{m=1}^{M}\right)=\sum_{m=1}^{M} \sum_{k=1}^{K} f\left(\alpha l_{k}\left(x_{m}\right) ; \alpha s_{k}\left(x_{m}\right)\right),
$$

where

$$
\mathbf{s}\left(x_{m}\right)=\left[s_{1}\left(x_{m}\right), \ldots, s_{K}\left(x_{m}\right)\right], \quad m=1, \ldots, M,
$$

and where $f(\cdot ; \cdot)$ is the Poisson log-likelihood:

$$
f(y ; \lambda)=-\lambda+y \log (\lambda)+\log (y !)
$$

As derived in the previous section, the position likelihood is:

$$
L_{2}\left(x_{p},\left\{\mathbf{s}\left(x_{m}\right)\right\}_{m=1}^{M}, \varepsilon\right)=\sum_{k=1}^{K} f\left(y_{k} ; \varepsilon s_{k}\left(x_{p}\right)\right) .
$$

Combining these two likelihood functions yields the following ML criterion:

$$
\hat{x}=\arg \max _{x_{p}} \max _{\mathbf{s}\left(x_{1}\right), \ldots, \mathbf{s}\left(x_{M}\right)} \max _{\varepsilon} \Phi_{1}\left(x_{p},\left\{\mathbf{s}\left(x_{m}\right)\right\}, \varepsilon\right),
$$

where

$$
\begin{aligned}
& \Phi_{1}\left(x_{p},\left\{\mathbf{s}\left(x_{m}\right)\right\}, \varepsilon\right)=L_{1}\left(\left\{\mathbf{s}\left(x_{m}\right)\right\}\right)+L_{2}\left(x_{p},\left\{\mathbf{s}\left(x_{m}\right)\right\}, \varepsilon\right) \\
& =L_{1}\left(\left\{\mathbf{s}\left(x_{m}\right)\right\}_{m=1}^{M}\right)+\sum_{k=1}^{K}\left[-\varepsilon s_{k}\left(x_{p}\right)+y_{k} \log \left(\varepsilon s_{k}\left(x_{p}\right)\right)\right] .
\end{aligned}
$$

Analytically maximizing over $\varepsilon$ yields

$$
\hat{\varepsilon}=\frac{\sum_{k=1}^{K} y_{k}}{\sum_{k=1}^{K} s_{k}\left(x_{p}\right)}
$$

so (7) simplifies to:

$$
\hat{x}=\arg \max _{x_{p}} \max _{\mathbf{s}\left(x_{1}\right), \ldots, \mathbf{s}\left(x_{M}\right)} \Phi_{2}\left(x_{p},\left\{\mathbf{s}\left(x_{m}\right)\right\}\right),
$$

where

$$
\begin{gathered}
\Phi_{2}\left(x_{p},\left\{\mathbf{s}\left(x_{m}\right)\right\}\right)=\sum_{m=1}^{M} \sum_{k=1}^{K} f\left(\alpha l_{k}\left(x_{m}\right) ; \alpha s_{k}\left(x_{m}\right)\right) \\
+\sum_{k=1}^{K} y_{k}\left[\log \left(s_{k}\left(x_{p}\right)\right)-\log \left(\sum_{j=1}^{K} s_{j}\left(x_{p}\right)\right)\right]
\end{gathered}
$$

excluding terms involving only $\left\{y_{k}\right\}$. Note that the last line of (8) is equivalent to (2). For $x_{m} \neq x_{p}$, there is 
no coupling between the LSF likelihood and the position likelihood, yielding:

$\hat{\mathbf{s}}\left(x_{m}\right)=\left[l_{1}\left(x_{m}\right), \ldots, l_{K}\left(x_{m}\right)\right], m=1, \ldots, p-1, p+1, \ldots M$.

Combining these terms with (8) yields

$$
\hat{x}=\arg \max _{x_{p}} \max _{\mathbf{S}\left(x_{p}\right)} \Phi_{3}\left(x_{p}, \mathbf{s}\left(x_{p}\right)\right),
$$

where

$$
\begin{gathered}
\Phi_{3}\left(x_{p}, \mathbf{s}\left(x_{p}\right)\right)=\sum_{m=1}^{M} \sum_{k=1}^{K} f\left(\alpha l_{k}\left(x_{m}\right) ; \alpha l_{k}\left(x_{m}\right)\right) \\
-f\left(\alpha l_{k}\left(x_{p}\right) ; \alpha l_{k}\left(x_{p}\right)\right)+f\left(\alpha l_{k}\left(x_{p}\right) ; \alpha s_{k}\left(x_{p}\right)\right) \\
+\sum_{k=1}^{K} y_{k}\left[\log \left(s_{k}\left(x_{p}\right)\right)-\log \left(\sum_{j=1}^{K} s_{j}\left(x_{p}\right)\right)\right] .
\end{gathered}
$$

The first double sum is independent of $x_{p}$, so we drop it, leaving:

$$
\hat{x}=\arg \max _{x_{p}} \max _{\mathbf{S}\left(x_{p}\right)} \Phi_{4}\left(x_{p}, \mathbf{s}\left(x_{p}\right)\right),
$$

where

$$
\begin{gathered}
\Phi_{4}\left(x_{p}, \mathbf{s}\left(x_{p}\right)\right)= \\
-\sum_{k=1}^{K}\left[-\alpha l_{k}\left(x_{p}\right)+\alpha l_{k}\left(x_{p}\right) \log \left(\alpha l_{k}\left(x_{p}\right)\right)-\log \left(\alpha l_{k}\left(x_{p}\right)\right) !\right] \\
+\sum_{k=1}^{K}\left[-\alpha s_{k}\left(x_{p}\right)+\alpha l_{k}\left(x_{p}\right) \log \left(\alpha s_{k}\left(x_{p}\right)\right)-\log \left(\alpha l_{k}\left(x_{p}\right)\right) !\right] \\
+\sum_{k=1}^{K} y_{k}\left[\log \left(s_{k}\left(x_{p}\right)\right)-\log \left(\sum_{j=1}^{K} s_{j}\left(x_{p}\right)\right)\right] .
\end{gathered}
$$

Maximizing over $\mathbf{s}\left(x_{p}\right)$ yields:

$$
\hat{s}_{k}\left(x_{p}\right)=\frac{l_{k}\left(x_{p}\right)+\frac{1}{\alpha} y_{k}}{1+\frac{1}{\alpha}\left(\sum_{j=1}^{K} y_{j}\right) /\left(\sum_{j=1}^{K} l_{j}\left(x_{p}\right)\right)} .
$$

Thus, the LSF parameter estimate is a weighted combination of the calibration data and the PMT outputs. Note that as $\alpha$ increases, $\hat{s}_{k}\left(x_{p}\right)$ approaches $l_{k}\left(x_{p}\right)$, as one expects. Substituting this expression into (9) yields:

$$
\hat{x}=\arg \max _{x_{p}} \Phi_{5}\left(x_{p}\right),
$$

where

$$
\begin{gathered}
\Phi_{5}\left(x_{p}\right)= \\
\sum_{k=1}^{K} \alpha l_{k}\left(x_{p}\right) \log \left(\frac{1+\frac{1}{\alpha} y_{k} / l_{k}}{\left.1+\frac{1}{\alpha}\left(\sum_{j=1}^{K} y_{j}\right) /\left(\sum_{j=1}^{K} l_{j}\left(x_{p}\right)\right)\right)}\right) \\
-\sum_{k=1}^{K} y_{k}\left[\log \left(1+\frac{1}{\alpha} \frac{\sum_{j=1}^{K} y_{j}}{\sum_{j=1}^{K} l_{j}\left(x_{p}\right)}\right)\right] \\
+\sum_{k=1}^{K} y_{k}\left[\log \left(l_{k}\left(x_{m}\right)+y_{k} / \alpha\right)-\log \left(\sum_{j=1}^{K} l_{j}\left(x_{m}\right)\right)\right] .
\end{gathered}
$$

Using the fact that $\log (x) \approx x-1$ for $x \approx 1$, one can show that the first line above is negligible. Also, if the PMT array has fairly uniform total sensitivity $\left(\sum_{k=1}^{K} l_{k}(x)\right.$ is nearly constant), then we can also ignore the second line. Thus, we have reduced this joint estimation problem to the following proposed estimator:

$$
\hat{x}=\arg \max _{x_{p}} \Phi\left(x_{p}\right)
$$

where

$\Phi\left(x_{p}\right)=\sum_{k=1}^{K} y_{k}\left[\log \left(l_{k}\left(x_{p}\right)+y_{k} / \alpha\right)-\log \left(\sum_{j=1}^{K} l_{j}\left(x_{p}\right)\right)\right]$.

Assuming each estimated LSF is smooth, one can implement this robust estimator by searching for a zero-crossing of:

$$
\sum_{k=1}^{K} y_{k}\left[\frac{l_{k}\left(x_{m+1}\right)-l_{k}\left(x_{m}\right)}{l_{k}\left(x_{m}\right)+y_{k} / \alpha}-\frac{\sum_{j=1}^{K}\left(l_{j}\left(x_{m+1}\right)-l_{j}\left(x_{m}\right)\right)}{\sum_{j=1}^{K} l_{j}\left(x_{m}\right)}\right] .
$$

Note that as $\alpha$ increases, this form approaches a discretized version of the original ML estimator (3).

Problems arise in the original ML method when an unusually large PMT output $y_{k}$ is magnified by a small LSF measurement $l_{k}(x)$. The additional term $y_{k} / \alpha$ in the denominator of this robust estimator diminishes the contribution of large measurements from the tails of the LSF, where the relative measurement errors are the largest.

The CR bound given by (5) is for the case when each LSF is known exactly. It would be desirable to have a bound that accounts for the uncertainty in the LSF measurements. This appears difficult because once one considers the fact that the calibration procedure produces a finite number of LSF measurements, the problem is then inherently discrete. Nevertheless, the expression (5) can still serve as a lower bound, albeit a possibly optimistic one.

Although (11) and (12) are incompatible with the hybrid position estimator circuit described in [2], one could easily implement either estimator using a digital signal processing chip. A two-dimensional implementation is also possible, such as the iterative approach described in [4].

\section{Simulation}

To evaluate the new estimator, we simulated one dimension of a position sensitive detector similar to those in the SPRINT II system [5]. The detector modules in this system are approximately $140 \mathrm{~mm}$ wide, and the position in a typical row is estimated from five PMT outputs. The five LSFs shown in Fig. 1 are similar to those for SPRINT II. The sensitivity of the detector module is such that the PMTs detect a total of approximately 700 photo-electrons per ${ }^{99 m} \mathrm{Tc}$ gamma photon scintillation. Therefore, in our simulation: $\sum_{k=1}^{K} s_{k}(x) \approx 700$. 
The CR lower bound for known LSF is shown in Fig. 2 . The FWHM resolution is approximately $3 \mathrm{~mm}$, agreeing with that reported in [2]. Fig. 2 also shows the analytical ideal ML standard deviation (4) for the known LSF case.

We have actually presented four position estimators that should be compared: the ordinary likelihood search given by (2), the ordinary zero-crossing search given by (3), the "robust" likelihood search given by (11), and the "robust" zero-crossing search given by (12). We denote these four approaches $\mathrm{S} 1, \mathrm{Z} 1, \mathrm{~S} 2$, and $\mathrm{Z} 2$ respectively.

To compare the four estimators, we chose $28 x$ positions spaced at $5 \mathrm{~mm}$ intervals, and generated 1000 realizations of the PMT outputs (1) and the LSF measurements (6) at each $x$ position. The LSF measurements were taken at $0.5 \mathrm{~mm}$ intervals. For each realization, the $\mathrm{S} 1$ and $\mathrm{S} 2$ estimates were performed by searching over the entire $140 \mathrm{~mm}$ field of view (FOV); the $\mathrm{Z} 1$ and $\mathrm{Z} 2$ estimates were found by searching choosing the left-most zero crossing in a $15 \mathrm{~mm}$ interval centered about the true position. For several realizations, no zero-crossing was found in the intervals for the three $x$ positions at the edges of the FOV. Therefore, in the remaining analysis we focus on the 22 central $x$ positions, at locations $17 \mathrm{~mm}, 22 \mathrm{~m}, \ldots, 122 \mathrm{~mm}$.

Tables I and II compare the performance of the four estimators to the CR bound (5). The tables report the number of $x$ positions (out of 22) for which the estimator's variance was significantly higher or lower than the CR bound (at $p=0.05$ using the $\chi^{2}$ statistic). It appears that all four estimators are nearly efficient even in the presence of noisy LSF measurements, except when $\alpha$ is very small.

Table III compares the performance of the two zerocrossing methods with the two corresponding likelihood search methods. The table reports the number of $x$ positions (out of 22) for which the zero-crossing methods have a significantly higher or lower variance than the likelihood search methods. (These were tested at $p=0.05$ using the F-test.) Despite the fact that the local search used for the zero-crossing method should have biased the results in favor of the zero-crossing method, we see from Table III that in fact the likelihood search methods outperform the zerocrossing methods when the LSF measurements are particularly poor. This is probably due to the fact that the zero-crossing approach cannot distinguish between global and local maxima. If the LSF measurements are very noisy (small $\alpha$ ), then local minima are more likely. However, for practical values of $\alpha$, there appears to be little difference between the performance of the zero-crossing methods and the likelihood search methods.

Finally, Table IV compares the performance of S1 with $\mathrm{S} 2$ and that of $\mathrm{Z1}$ with Z2, again using the F-test. Apparently the original estimators given by (2) and (3) are fairly robust to Poisson errors in the measured LSFs, since there appears to be no significant difference between $\mathrm{S} 1$ and $\mathrm{S} 2$ or $\mathrm{Z} 1$ and $\mathrm{Z} 2$.

\section{DISCUSSION}

As mentioned in the introduction, this project was an attempt to reduce image artifacts that we attributed to systematic errors in position estimation. We hypothesized that these errors were due in part to the Poisson uncertainty in the LSF measurements. The simulation results described above do not support this hypothesis. The artifacts remain though, and with the benefit of hindsight it appears that the other sources of LSF measurement error are more important than the Poisson uncertainty. In particular, the quantization errors in the $A / D$ converter and the effects of background events are likely candidates since they have the largest relative effect near the tails of the LSF. Note that once one accounts for these sources of error, $\alpha$ is no longer proportional to the length of the time interval used for the calibration.

The joint estimation method described in Section II has applications beyond the position estimation problem. In particular, Clinthorne has proposed in [6] applying a similar, idea to improve emission image reconstruction in PET by accounting for the noise in the transmission scan.

Future work needs to focus on the other sources of LSF measurement errors. A LSF measurement model that accounts for those errors may be less amenable to the type of analytical maximization derived in Section II. Nevertheless, by exploiting the fact that LSFs are typically smooth functions of position, a more robust position estimation method should be achievable. 


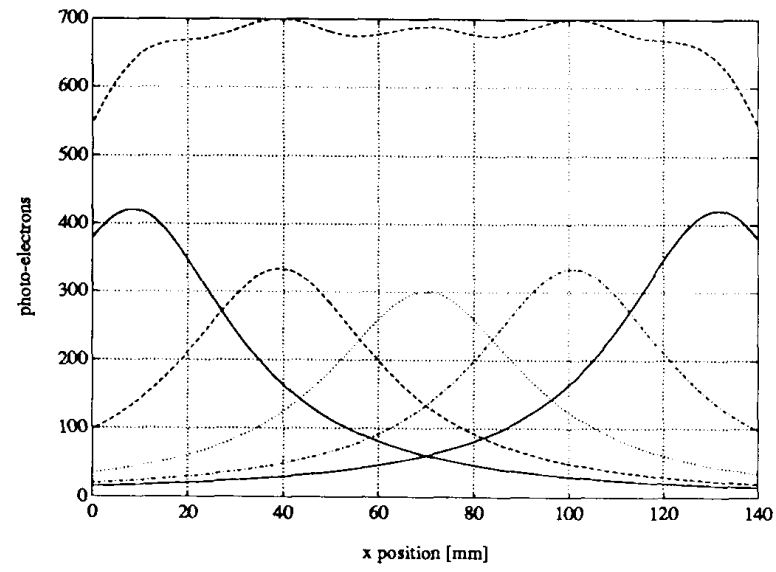

Figure 1: Simulated light spread functions (LSF) for five PMTs versus position. The top curve is the total light collection.

\section{REFERENCES}

[1] R. M. Gray and A. Macovski, "Maximum a posteriori estimation of position in scintillation cameras," IEEE Transactions on Nuclear Science, vol. 23, pp. 849-852, Feb. 1976.

[2] N. H. Clinthorne, W. L. Rogers, L. Shao, and K. F. Koral, "A hybrid maximum likelihood position computer for scintillation cameras," IEEE Transactions on Nuclear Science, vol. 34, pp. 97-101, Feb. 1987.

[3] B. Efron, The Jackknife, the Bootstrap and Other Resampling Plans. Philadelhpia, PA: SIAM, 1982.

[4] X. Liu, N. H. Clinthorne, L. Shao, K. F. Koral, and W. L. Rogers, "Recursive calculation of the twodimensional maximum likelihood position estimate for a scintillation camera," IEEE Transactions on Nuclear Science, vol. 37, pp. 713-717, Apr. 1990.

[5] W. L. Rogers, N. H. Clinthorne, L. Shao, P. Chiao, Y. Ding, J. A. Stamos, and K. F. Koral, "SPRINT II: A second generation single photon ring tomograph," IEEE Transactions on Medical Imaging, vol. 7, pp. 291-297, Dec. 1988.

[6] N. H. Clinthorne, J. A. Fessler, G. D. Hutchins, and W. L. Rogers, "Joint maximum likelihood estimation of emission and attenuation data in PET," 1991. To be presented at Nuclear Science Symposia.

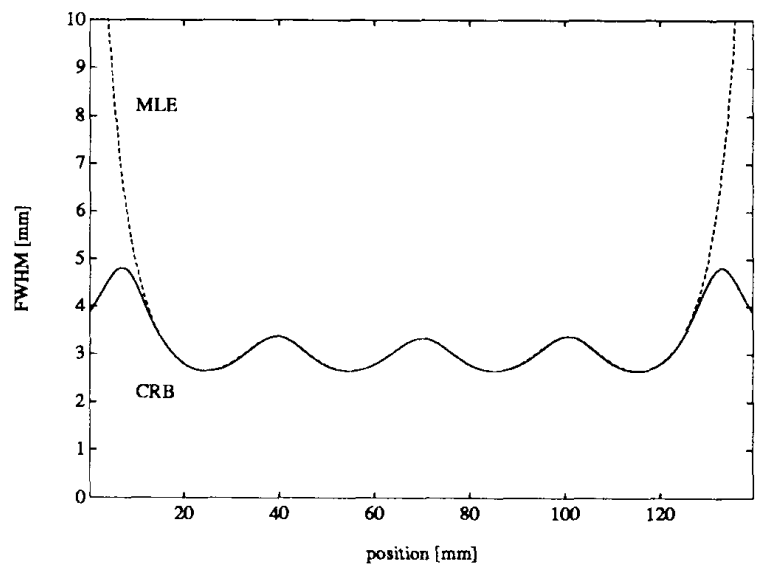

Figure 2: CR bound for position estimation (solid) and standard deviation of ML estimator for known LSF.

\begin{tabular}{|l||c|c||c|c||}
\hline$\alpha$ & $\sigma_{\mathrm{S} 1}^{2}>\sigma_{\mathrm{CR}}^{2}$ & $\sigma_{\mathrm{S} 1}^{2}<\sigma_{\mathrm{CR}}^{2}$ & $\sigma_{\mathrm{Z} 1}^{2}>\sigma_{\mathrm{CR}}^{2}$ & $\sigma_{\mathrm{Z} 1}^{2}<\sigma_{\mathrm{CR}}^{2}$ \\
\hline $10^{4}$ & 3 & 1 & 4 & 0 \\
$10^{3}$ & 3 & 0 & 8 & 0 \\
$10^{2}$ & 16 & 0 & 22 & 0 \\
\hline
\end{tabular}

Table I: Comparing the "ordinary" ML methods with CR bound (number significant at $\mathrm{p}=0.05$ out of 22 possible).

\begin{tabular}{|l||c|c||c|c||}
\hline$\alpha$ & $\sigma_{\mathrm{S} 2}^{2}>\sigma_{\mathrm{CR}}^{2}$ & $\sigma_{\mathrm{S} 2}^{2}<\sigma_{\mathrm{CR}}^{2}$ & $\sigma_{\mathrm{Z} 2}^{2}>\sigma_{\mathrm{CR}}^{2}$ & $\sigma_{\mathrm{Z} 2}^{2}<\sigma_{\mathrm{CR}}^{2}$ \\
\hline $10^{4}$ & 1 & 1 & 4 & 0 \\
$10^{3}$ & 1 & 0 & 8 & 0 \\
$10^{2}$ & 15 & 0 & 22 & 0 \\
\hline
\end{tabular}

Table II: Comparing the "robust" ML methods with CR bound.

\begin{tabular}{|l||c|c||c|c||}
\hline$\alpha$ & $\sigma_{\mathrm{Z} 1}^{2}>\sigma_{\mathrm{S} 1}^{2}$ & $\sigma_{\mathrm{Z} 1}^{2}<\sigma_{\mathrm{S} 1}^{2}$ & $\sigma_{\mathrm{Z} 2}^{2}>\sigma_{\mathrm{S} 2}^{2}$ & $\sigma_{\mathrm{Z} 2}^{2}<\sigma_{\mathrm{S} 2}^{2}$ \\
\hline $10^{4}$ & 3 & 2 & 4 & 1 \\
$10^{3}$ & 2 & 1 & 2 & 1 \\
$10^{2}$ & 22 & 0 & 22 & 0 \\
\hline
\end{tabular}

Table III: Comparing the variance of the likelihood-search methods with the zero-crossing methods.

\begin{tabular}{|l||c|c||c|c||}
\hline$\alpha$ & $\sigma_{\mathrm{S} 2}^{2}>\sigma_{\mathrm{S} 1}^{2}$ & $\sigma_{\mathrm{S} 2}^{2}<\sigma_{\mathrm{S} 1}^{2}$ & $\sigma_{\mathrm{Z} 2}^{2}>\sigma_{\mathrm{Z} 1}^{2}$ & $\sigma_{\mathrm{Z} 2}^{2}<\sigma_{\mathrm{Z} 1}^{2}$ \\
\hline $10^{4}$ & 0 & 0 & 0 & 0 \\
$10^{3}$ & 0 & 0 & 0 & 0 \\
$10^{2}$ & 0 & 2 & 0 & 0 \\
\hline
\end{tabular}

Table IV: Comparing the variance of the "robust" methods with the "ordinary" methods. 